\title{
Análise da estrutura e do estoque de fitomassa de uma floresta secundária da região de Manaus AM, dez anos após corte raso seguido de fogo
}

\author{
Adriano José Nogueira LIMA ${ }^{1}$, Liliane Marins TEIXEIRA ${ }^{1}$, Vilany Matilla Colares CARNEIRO ${ }^{1}$, Joaquim \\ dos SANTOS $^{1}$, Niro HIGUCHI ${ }^{1}$
}

\begin{abstract}
RESUMO
$\mathrm{Na}$ Amazônia, o fogo é ainda o principal trato cultural utilizado no preparo de solo para agricultura e pecuária, tanto pelos pequenos como pelos grandes fazendeiros. Combinando à baixa fertilidade do solo e ao baixo preço da terra, assim que as fontes naturais de nutrientes são exauridas, as áreas são abandonadas e novas florestas primárias são derrubadas e queimadas. Por conta disso, grandes extensōes de área da Amazônia são cobertas por florestas secundárias originadas de áreas abandonadas pela agricultura ou pastagem. Este estudo foi conduzido em uma área experimental usada em uma pesquisa sobre eficiência de combustão e emissão de gás carbônico da floresta amazônica, localizada aproximadamente $50 \mathrm{~km}$ ao norte de Manaus. A vegetação da área experimental foi derrubada e queimada em 1991, simulando as condições em que o pequeno agricultor prepara o solo para plantios de subsistência. Dez anos após a queimada, a floresta secundária ainda é bastante diferente da floresta original. As espécies vegetais dominantes são, principalmente, das famílias botânicas Annonaceae, Arecaceae, Burseraceae, Cecropiaceae, Euphorbiaceae, Fabaceae, Lecythidaceae, Melastomataceae, Mimosaceae, Sapindaceae e Sterculiaceae. O estoque de biomassa recuperado, dez anos após a formação da capoeira estudada, é de aproximadamente $16 \%$, ou seja, a capoeira apresenta um estoque médio de 56,2 t.ha ${ }^{-1} \pm 12$ (IC $95 \%$ ), enquanto que o estoque da floresta primária é de 339,7 th.ha-1 \pm 66,7 (IC 95\%).
\end{abstract}

PALAVRAS-CHAVE

Estrutura horizontal, sucessão florestal, fogo, biomassa seca

\section{Biomass stock and structural analysis of a secondary forest in Manaus (AM) region, ten years after clear cutting followed by fire}

\begin{abstract}
In Amazonia, fire is widely used for soil preparation in the agriculture and cattle ranching, either by small or big farmers. The combination of low fertility of the soil and low price of the land usually leads to typical shift cultivation as soon as the natural sources of nutrients are exhausted, when new primary forests are felled and burned. Due to that, secondary forests originated from abandoned agriculture or pasture activity projects cover huge areas in the Brazilian Amazônia. This study was carried out in the Tropical Forestry Experimental Station (EESTIINPA), around 50 kilometers north of Manaus, the capital of Amazonas State. A secondary forest ("capoeira"), 10 years old, was selected for this study; its land use history includes clear cutting followed by fire. Two different samples of primary forests were included as control plots for the "capoeira". All individuals with diameter at breast height (DBH) greater than $5 \mathrm{~cm}$ were measured and recorded in a single area of 0,4 ha for each treatment. Ten years after burning, the secondary forest is still very different in comparison with primary forest, in terms of important parameters of the vegetation structure, such as floristic composition, abundance, frequency, dominance, index of value of importance (IVI) and the diameter distribution. In "capoeira" studied the dominant species are mainly of the following botanical families: Annonaceae, Arecaceae, Burseraceae, Cecropiaceae, Euphorbiaceae, Fabaceae, Lecythidaceae, Melastomataceae, Mimosaceae, Sapindaceae and Sterculiaceae. The estimates of dry biomass were 56.2 t. ha $a^{-1} \pm 12$ (CI 95\%) and 339.7 t.ha \pm 66.7 (CI $\left.95 \%\right)$ for "capoeira" and primary forest, respectively.
\end{abstract}

KEY-WORDS

Horizontal structure, forest succession, fire, dry biomass

1'Instituto Nacional de Pesquisas da Amazônia, Coordenação de Pesquisas em Ciências em Silvicultura Tropical - CPST. e-mail: adriano@inpa.gov.br 


\section{INTRODUÇÃO}

Nas regiōes tropicais, a ação antrópica tem provocado o corte raso de florestas primárias para projetos de desenvolvimento como agricultura, pecuária e mineração. No caso da agropecuária, a prática mais comum é a derruba-e-queima ("slash-and-burn"), seguido de cultivo itinerante (shift cultivation). Por conta disso, excetuando a região amazônica, as paisagens das regiōes tropicais vêm sendo dominadas por capoeiras (Gómez-Pompa; VásquezYanes, 1974; Brown \& Lugo, 1990). Na maioria dos países, principalmente aqueles com altas densidades populacionais, as capoeiras têm sido usadas e reutilizadas, tanto para a agricultura itinerante, como também para o fornecimento de matéria-prima às indústrias madeireiras.

$\mathrm{Na}$ Amazônia brasileira, as florestas primárias ainda são predominantes. No entanto, a velocidade do desmatamento é motivo de preocupação de todos os segmentos da sociedade. Até 2005, o desmatamento acumulado na Amazônia é de 651.395 $\mathrm{km}^{2}$ ou aproximadamente 65 milhōes de hectares, enquanto que somente no Estado do Amazonas tem-se aproximadamente 30 mil km² ou 3 milhões de hectares. Ao contrário de outros países tropicais, na Amazônia, $85 \%$ das áreas desmatadas anualmente são superiores a 15 ha (INPE, 2001), não caracterizando uma prática típica de cultivo itinerante por pequenos produtores. Por outro lado, independentemente do tamanho do desmatamento, o trato cultural mais utilizado na limpeza do terreno é a queimada. Em relação aos outros países tropicais, o Brasil tem uma situação bastante confortável: há uma área de capoeira muito grande para uma população relativamente pequena (aproximadamente 2 ha per capita) e ainda $85 \%$ de sua cobertura florestal original, equivalente a mais de 300 milhôes de hectares, ainda intacta. O desmatamento da Amazônia não a fez mais rica e nem a ser o celeiro do mundo, como se preconizava durante o milagre econômico. Atualmente, o bom senso indica o aproveitamento das capoeiras e a preservação das florestas primárias.

Para aproveitar as capoeiras é preciso, antes de tudo, entender a sua dinâmica, considerando os diferentes usos do solo (agricultura extensiva, pastagem, cultivo itinerante e mineração) e a intensidade e a duração dos mesmos. Temos na Amazônia, capoeiras de várias idades, originadas de diferentes usos e diferentes tamanhos. De um modo geral, de acordo com Mesquita (2000), há vários estudos sobre a dinâmica de clareiras, em diferentes sítios como Sul do Pará, Acre, Rondônia e região de Manaus.

De certa forma, as informações existentes já podem ser usadas para modelagem da dinâmica, fazer simulaçōes considerando diferentes cenários e pressupostos e planejar medidas necessárias para o adequado manejo das capoeiras. Em todos os estudos já realizados, as capoeiras foram formadas depois de abandonadas por algum tipo de uso do solo. Para modelagem é importante ter uma verdade de campo que contemple uma situação onde a capoeira foi formada depois de derruba-e-queima da floresta primária, sem nenhum uso do solo. Este é o nosso caso e, talvez, com os incêndios florestais ocorrendo naturalmente em florestas primárias na Amazônia, teremos um novo modelo de capoeiras.

O objetivo deste trabalho é analisar a estrutura da floresta secundária (capoeira), considerando apenas as espécies arbóreas, dez anos após a derruba-e-queima. Esta área foi abandonada após a pesquisa sobre eficiência de combustão e emissão de gás carbônico da floresta amazônica, ou seja, sem nenhum uso do solo. Foi usada como testemunha, uma área de mesmo tamanho, coberta com floresta primária, praticamente contígua à capoeira estudada. A análise estrutural foi executada em termos de estrutura horizontal da floresta, de acordo com Jardim \& Hosokawa (1987), de modo a possibilitar a análise das características ecológicas, sinecológicas e a dinâmica da floresta. Do ponto de vista quantitativo, foi usada, principalmente, a acumulaçāo da biomassa acima do nível do solo, dez anos após o abandono da área, para se ter uma idéia do tempo necessário para capoeira retornar ao estoque original.

\section{MATERIAL E MÉTODOS}

\section{LOCALIZAÇÃO E CARACTERIZAÇÃO DA ÁREA}

A área de estudo fica localizada em terras da Estação Experimental de Silvicultura Tropical do Instituto Nacional de Pesquisas da Amazônia (EEST/INPA), no Distrito Agropecuário da Superintendência da Zona Franca de Manaus - SUFRAMA, no quilômetro $45 \mathrm{da}$ BR-174. As coordenadas geográficas da Estação são $02^{\circ} 35^{\prime} 55,5^{\prime \prime}$ de latitude sul e 60 $02^{\circ} 14,8^{\prime \prime}$ de longitude oeste Grw. A EEST limita-se ao norte com terras da Comissão Executiva do Plano da Lavoura Cacaueira - CEPLAC; ao sul com terras do Instituto Brasileiro do Meio Ambiente e dos Recursos Naturais Renováveis (IBAMA).

A pesquisa foi feita na Estação Experimental de Silvicultura Tropical - EEST/S-8, cerca de um quilômetro a noroeste da Estação, com acesso via Igarapé do Guaraná ou pelo ramal da CEPLAC, $2 \mathrm{~km}$ ao norte da sede. O solo do local, segundo Ranzani (1980) é Latossolo amarelo de textura argilosa. Segundo ainda o mesmo autor, a S-8 se caracteriza pela presença de platôs típicos com diâmetros médios de $500 \mathrm{~m}$. O clima é quente e úmido com precipitaçóes elevadas que tendem a compensar a existência de uma estação seca, embora não acentuada; tipo climático é Am na classificação de Köppen (Ranzani, 1980). A precipitação média anual é de $2.500 \mathrm{~mm}$. A temperatura média anual é de $26,7^{\circ} \mathrm{C}$. O período entre julho e outubro-novembro a evapotranspiração potencial é superior à precipitação. A vegetação é caracterizada como mata de terra-firme sendo distinguida por uma estrutura relativamente grande: o dossel atinge alturas de $18-30 \mathrm{~m}$, com emergentes superiores a $40 \mathrm{~m}$. O chão caracteriza-se por uma manta mais fina de raízes e há pouca 
acumulação de serapilheira. Em termos de composição florística, as famílias mais importantes são Leguminosae, Sapotaceae e Lecythidaceae (Ranzani, 1980).

\section{CARACTERIZAÇÃO DOS DADOS}

Os dados foram coletados na $\mathrm{S}-8$, em duas cronosseqüências distintas: floresta primária e capoeira de dez anos formada depois da "derrubada-e-queima" da floresta primária. Para a preparação da área que hoje é uma capoeira, um quadrado de um hectare de floresta primária foi derrubado em julho de 1991 e queimado em novembro do mesmo ano. Essa área foi originalmente desenhada para os estudos sobre eficiência de combustão e emissão de gás carbônico da floresta amazônica (Carvalho Jr et al., 1995).

Para ter uma idéia da dinâmica da capoeira estudada, foi tomado como comparador um quadrado de mesmo tamanho de floresta primária, praticamente contígua à capoeira. Nos dois sítios estudados, foram alocadas aleatoriamente 10 unidades amostrais de $20 \times 20 \mathrm{~m}$, para a coleta de dados. Em cada parcela, todos os indivíduos com diâmetro à altura do peito (DAP) maior ou igual a $5 \mathrm{~cm}$ foram medidos e identificados preliminarmente com os nomes comuns.

\section{ESTIMATIVAS DOS PARÂMETROS DA ESTRUTURA FLORESTAL}

A estrutura horizontal foi analisada pelo índice de valor de importância (IVI), que inclui os índices de abundância (Ab), freqüência (F) e dominância (Do), segundo Montoya Maquim (1966), Cain \& Castro (1959), Lamprecht (1964) e Finol (1971).

A biomassa seca $\left(\mathrm{t} \cdot \mathrm{ha}^{-1}\right)$ para floresta secundária foi estimada, separadamente, para a espécie Cecropia sciadophylla Mart. e para um grupo de espécies de capoeira, usando as seguintes equaçóes desenvolvidas por Nelson et al. (1999), sendo:

Ln P $=-2,5118+2,4257$.Ln DAP ( Cecropia sciadophylla Mart.) Ln $\mathrm{P}=-1,9968+2,4128$. Ln DAP (Grupo de espécies) sendo:

Ln = logaritmo natural;

$\mathrm{P}=$ peso seco da biomassa em $\mathrm{kg}$

$\mathrm{DAP}=$ diâmetro à altura do peito em $\mathrm{cm}$;

A biomassa da floresta primária foi estimada usando as equações desenvolvidas por Higuchi et al. (1998), separadamente, para indivíduos com DAP entre 5 e $20 \mathrm{~cm}$ e para indivíduos com DAP e" $20 \mathrm{~cm}$, da seguinte maneira:
Ln $P F=-1,754+2,665 . \mathrm{Ln}$
$\operatorname{DAP}(5 \leq \mathrm{DAP}<20 \mathrm{~cm})$

$$
\left[\mathrm{R}_{\mathrm{a}}^{2}=0,92 \text { e } \mathrm{S}_{\mathrm{yx}}=0,43\right]
$$
Ln PF $=-0,151+2,17$. Ln DAP
$\operatorname{DAP}(\geq 20 \mathrm{~cm})$

sendo:

$\mathrm{PF}=$ peso fresco em $\mathrm{kg}$

Para o peso seco foi estimado usando a concentração de água (igual a 40\%) determinada por Higuchi \& Carvalho Jr. (1994).

\section{RESULTADOS E DISCUSSÃO}

\section{COMPOSIÇÃO FLORESTAL}

Na capoeira foram observados 459 indivíduos arbóreos com $\mathrm{DAP} \geq 5 \mathrm{~cm}$, distribuídos em 34 famílias botânicas e 53 espécies diferentes, considerando todas as 10 unidades amostrais (4000 $\mathrm{m}^{2}$ ). Neste levantamento, as famílias que mais se destacaram em ordem decrescente por número de espécies foram: Annonaceae com cinco espécies, Melastomataceae e Mimosaceae com quatro espécies. Outras famílias também ocorreram, porém contribuíram apenas com duas espécies.

$\mathrm{Na}$ floresta primária (testemunha), foram encontrados 532 indivíduos arbóreos com $\mathrm{DAP} \geq 5 \mathrm{~cm}$, distribuídos em 41 famílias botânicas e 138 espécies diferentes, em $4000 \mathrm{~m}^{2}$. Nessa floresta, as famílias que mais se destacaram por número de espécies foram: Sapotaceae (16), Chrysobalanaceae, Lecythidaceae e Mimosaceae (8) seguidas por Annonaceae, Caesalpiniaceae e Lauraceae (7).

\section{ÍNDICE DE VALOR DE IMPORTÂNCIA (IVI)}

O IVI das 15 espécies mais importantes são apresentados na Tabela 1A (capoeira) e Tabela 1B (floresta primária). As 15 espécies listadas na Tabela $1 \mathrm{~A}$ representam $82 \%$ do IVI de todas as espécies presentes na capoeira, enquanto que, na floresta primária, as 15 espécies representam apenas 32\%. No entanto, do ponto de vista de diversidade e dominância, a capoeira ainda está muito distante da floresta primária. Esta evidência encontrada para a vegetação arbórea é praticamente inversa ao da mesofauna do solo que, segundo Antony (1997), cinco anos após a formação da mesma capoeira, as diversidades (grupos taxonômicos) das duas cronosseqüências eram as mesmas, porém a abundância da capoeira representava apenas 59\%.

Somente ingá branco (Inga sp.) faz parte das 15 espécies com maior IVI nas duas cronosseqüências, sendo que as demais espécies são exclusivas de cada cronosseqüência. Na capoeira, a presença de embaúba gigante (Cecropia sciadophylla Mart. Cecropiaceae), dima (Croton lanjouwensis Jabl. - Euphorbiaceae), piriquiteira amarela (Laetia procera (Poepp.) Eichler. Flacourtiaceae), murici da mata (Byrsonima crispa A. Juss. Malpighiaceae) e as espécies do gênero Vismia (Clusiaceae), são marcantes na paisagem da capoeira estudada.

Essas espécies praticamente não são encontradas na floresta primária, demonstrando que são espécies especialistas em capoeiras. Esses resultados são compatíveis com os de Nelson et al. (1999). Na floresta primária, a distribuição das espécies é 
mais uniforme, sem uma predominância destacada de uma ou outra espécie.

Tabela 1A - Índices de Valor de Importância - IVI, absoluto e relativo, referente à vegetação na área de capoeira.

\begin{tabular}{lrcc}
\hline \multicolumn{1}{c}{ Espécie } & IVI & IVI(\%) \\
\hline Embaúba gigante (Cecropia sciadophylla Mart.) & 69,01 & 23,00 \\
Dima (Croton lanjouwensis Jabl.) & 26,94 & $\mathbf{8 , 9 8}$ \\
Piriquiteira amarela (Laetia procera (Poepp.) Eicher) & 19,46 & 6,49 \\
Murici da mata (Byrsonima crispa A. Juss.) & 18,89 & 6,30 \\
Lacre branco (Vismia cayennensis (Jacq.) Pers.) & 18,24 & 6,08 \\
Faveira camuzé (Stryphnodendron guianense (Aubl.) Benth.) & 15,01 & 5,00 \\
Ingá branca (Inga parensis Ducke.) & 11,09 & 3,70 \\
Lacre vermelho (Vismia guianensis (Aubl.) Pers.) & 11,09 & $\mathbf{3 , 7 0}$ \\
Breu manga (Tetragastris altissima (Aubl.) Swart) & 11,00 & 3,67 \\
Tabaco bravo (Aegiphila sp. - Verbenaceae) & 9,83 & 3,28 \\
Morototó (Schefflera morototoni (Aubl.) Maguire, Steyerm. \& Frodin) & 9,67 & 3,22 \\
Sacaca brava (Croton draconoides Mull. Arg.) & 7,29 & 2,43 \\
Envira fofa (Guatteria discolor R.E. Fr.) & 7,14 & 2,38 \\
Envira bobó (Rollinia exsucca (DC. ex Dunal) A. DC.) & 7,06 & $\mathbf{2 , 3 5}$ \\
Buxuxu orelha de burro (Schomburgkia fimbriata (Kunth) Hoehne) & 4,42 & 1,47 \\
TOTAL & 82,05 \\
\hline
\end{tabular}

Tabela 1B - Índices de Valor de Importância - IVI, absoluto e relativo, referente à vegetação na área de floresta primária.

\begin{tabular}{lcc}
\hline \multicolumn{1}{c}{ Espécie } & IVI & IVI(\%) \\
\hline Macucu chiador (Licania oblongifolia Standl.) & 9,49 & 3,16 \\
Abiurana bacuri (Chrysophyllum guyanense Klotzsch ex Miq.) & 8,80 & 2,93 \\
Urucurana (Sloanea floribunda Spruce ex Benth.) & 8,71 & 2,90 \\
Breu branco (Hemicrepidospermum rhoifolium (Benth.) Swart) & 7,11 & 2,70 \\
Breu vermelho (Protium apiculatum Swart) & 6,78 & 2,26 \\
Ripeiro preto (Eschweilera odora (Poepp. ex O. Berg) Miers) & 6,54 & 2,18 \\
Ingarana (Pithecelobium duckei Huber) & 6,24 & 2,08 \\
Mandioqueira (Qualea paraensis Ducke) & 6,06 & 2,02 \\
Pajurazinho (Licania kunthiana Hook. f.) & 5,80 & 1,93 \\
Matamatá amarelo (Eschweilera coriacea (DC.) S. A. Mori) & 5,80 & 1,93 \\
Louro preto (Ocotea neesiana (Miq.) Kosterm.) & 5,04 & 1,68 \\
Muirapiranga f. grande (Eperua bijuga Mart. ex Benth.) & 4,96 & 1,65 \\
Tapura (Tapura amazonica Poepp.) & 4,82 & 1,61 \\
Ingá branca (Inga paraensis Ducke) & 4,59 & 1,53 \\
Jaraí (Glycoxylon pedicellatum Ducke) & 4,48 & 1,49 \\
TOTAL & & 32,05 \\
\hline
\end{tabular}

\section{BIOMASSA SECA ACIMA DO NÍVEL DO SOLO}

Neste caso, foram incluídas na análise, as medições feitas na mesma capoeira, em 1998, usando parcelas temporárias. A Tabela 2 apresenta as estimativas de biomassa da capoeira (mediçōes de 1998 e 2001) e da floresta primária. Os estoques médios de biomassa das cronossequiências 1998, 2001 e floresta primária são respectivamente 49,0 t.ha ${ }^{-1} \pm 10,6$ (IC 95\%), 56,2 t.ha ${ }^{-1} \pm$ 12 (IC 95\%) e 339,7 t.ha ${ }^{-1} \pm 66,7$ (IC 95\%). O estoque de biomassa, dez anos após a formação da clareira, representa aproximadamente $16 \%$ do estoque original (floresta primária).

O incremento médio anual em biomassa, obtido em 1998, é de 7 t.ha ${ }^{-1} \cdot$ ano $^{-1}$ e cai para 5,6 t.ha ${ }^{-1} \cdot$ ano $^{-1}$, em 2001. A queda de produção no período de 3 anos é visível. O importante é continuar monitorando essa capoeira para ter uma idéia melhor sobre o tempo necessário para a capoeira voltar ao estoque original (da floresta primária). Fazendo uma projeção linear usando apenas o incremento de 2001, o tempo de retorno é de 50,9 anos \pm 4,6 (IC 95\%). Esses incrementos são baixos quando comparados aos de floresta primária e de florestas manejadas experimentalmente sob diferentes intensidades de corte (baixo, médio e alto), que segundo Higuchi et al. (1997) são 2,4 t.ha-

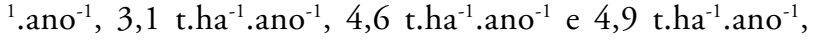
respectivamente. No entanto, comparando com outras capoeiras com diferentes históricos de uso do solo, as estimativas da nossa capoeira são maiores. Na Fazenda Monte Verde, por exemplo, situada no município de Peixe-Boi, micro-região Bragantina, os incrementos anuais de biomassa de capoeiras de 5,10 e 20 anos, são 2,6 t.ha ${ }^{-1} \cdot$ ano $^{-1}, 4,4 \mathrm{t} \cdot \mathrm{ha}^{-1} \cdot \mathrm{ano}^{-1}$ e $4 \mathrm{t} \cdot \mathrm{ha}^{-1} \cdot \mathrm{ano}^{-1}$, respectivamente (Salomão et al., 1998). Uhl (1982) demonstra que o tempo necessário para a recomposição de uma área desmatada depende de como a floresta foi removida e do tipo e

Tabela 2 - Estimativa de biomassa seca (t.ha-1) de três cronossequências (1998, 2001 e floresta primária) e tempo (anos) de retorno necessário ao estoque original.

\begin{tabular}{ccccc}
\hline Amostra & $\mathbf{1 9 9 8}$ & $\mathbf{2 0 0 1}$ & Original & Retorno \\
\hline 1 & 23,2 & 37,3 & 191,4 & 41,4 \\
2 & 28,8 & 38,4 & 203,8 & 43,0 \\
3 & 43,5 & 41,3 & 294,7 & 61,3 \\
4 & 43,6 & 45,2 & 317,7 & 60,3 \\
5 & 43,9 & 50,3 & 322,6 & 54,1 \\
6 & 44,6 & 51,6 & 324,9 & 53,0 \\
7 & 53,1 & 54,3 & 325,6 & 50,0 \\
8 & 64,0 & 64,6 & 428,2 & 56,3 \\
9 & 66,2 & 87,8 & 443,7 & 40,5 \\
10 & 79,0 & 91,6 & 544,4 & 49,5 \\
Média & 49,0 & 56,2 & 339,7 & 50,9 \\
Desvio padrão & 17,1 & 19,4 & 107,6 & 7,5 \\
IC ( $p=0,05)$ & $49,0 \pm 10,6$ & $56,2 \pm 12,0$ & $339,7 \pm 66,7$ & $50,9 \pm 4,6$ \\
\hline
\end{tabular}

do tempo de uso após a remoção, bem como do tamanho da clareira artificial aberta e da distância à floresta primária. Saldarriaga et al. (1988) estimaram que para as áreas agrícolas alcançassem área basal e biomassa semelhante às de florestas maduras seriam necessários, aproximadamente, 190 anos.

\section{CONCLUSÕES}

O estoque de biomassa recuperado, dez anos após a formação da capoeira estudada, é de aproximadamente $16 \%$, ou seja, a capoeira apresenta um estoque médio de 56,2 t.ha $^{-1} \pm 12$ (IC $95 \%)$, enquanto que o da floresta primária é de 339,7 t.ha ${ }^{-1} \pm$ 66,7 (IC 95\%). Fazendo-se uma projeção linear baseada no 
acúmulo de biomassa nos primeiros dez anos, serão ainda necessários mais 51 anos para que a capoeira estudada retorne ao seu estoque original (da floresta primária). Em capoeiras com históricos diferentes de uso do solo, a estimativa é de 190 anos.

Do ponto de vista de abundância de indivíduos arbóreos, esta capoeira já recuperou $84 \%$ do correspondente à floresta primária. No entanto, em relação à diversidade de espécies florestais, a capoeira ainda está muito aquém da floresta primária, ou seja, a capoeira tem apenas $39 \%$ das espécies que fazem parte da composição florística da floresta primária.

A paisagem da capoeira estudada é dominada por espécies como embaúba gigante ( Cecropia scyadophylla Mart. Cecropiaceae), dima (Croton lanjouwensis Jablonski Euphorbiaceae), piriquiteira amarela (Laetia procera (Poepp.) Eichler. - Flacourtiaceae), murici da mata ( Byrsonima crispa A. Juss. -Malpighiaceae) e as espécies do gênero Vismia (Clusiaceae). Essas espécies praticamente são ausentes na floresta primária, demonstrando que são espécies especialistas em clareiras. $\mathrm{Na}$ floresta primária, a distribuição das espécies é mais uniforme, sem uma predominância destacada de uma ou outra espécie.

\section{BIBLIOGRAFIA CITADA}

Antony, L.M.K. 1997. Abundância e distribuição vertical da fauna do solo de ecossistemas amazônicos naturais e modificados. In: BIONTE - Relatório Final. p. 249-255.

Brown, N.; Lugo, A.E. 1990. Tropical secundary forests. Journal of Tropical Ecology, 6: 1-32.

Cain, S.A.; Castro, G.M.O. 1959. Manual of vegetation analysis. Hafner Publ. Co., New York. 325pp.

Carvalho Jr, J.A.; Santos, J.M.; Santos, J.C.; Leitão, M.M.; Higuchi, N. 1995. A tropical rainforest clearing experiment by biomass burning in the Manaus region. Atmospheric Environment, 29(17): 2301-2309.

Finol, U.H. 1971. Nuevos parametros a consideraise en la analisis estructural de las selvas virgenes tropicales. Rev. For. Venezoelana, 14(21): 29-42.

Gómez-Pompa, A.; Vázquez-Yanes, C. 1974. Studies on the secundary sucession of tropical lowlands: The life cycle of secundary species. In: Proceedings, 1 st International Congress of Ecology, The Hague, The Netherlands.

Higuchi, N.; Carvalho Jr., J.A. 1994. Fitomassa e Conteúdo de Carbono de Espécies Arbóreas da Amazônia. In: Anais do Seminário "Emissão x Seqüestro de CO2 - Uma Nova Oportunidade de Negócios para o Brasil”. p. 127-153.
Higuchi, N.; Santos, J.; Ribeiro, R.J.; Freitas, J.V.; Vieira, G.; Coic, A.; Minette, L.J. 1997. Crescimento e incremento de uma floresta amazônica de terra-firme manejada experimentalmente. In: BIONTE - Relatório Final. p. 89-132.

Higuchi, N.; Santos, J.; Ribeiro, R.J.; Minette, L.; Biot, Y. 1998. Biomassa da parte aérea da vegetação da floresta tropical úmida de terra-firme da Amazônia Central. Acta Amazonica, 28(2): 153-166.

INPE. 2001. Desflorestamento na Amazônia, (www.inpe.gov.br).

INPE. 2006. Desflorestamento na Amazônia, (www.inpe.gov.br). Acesso: 31/07/06.

Jardim, F.C.S.; Hosokawa, R.T. 1987. Estrutura da floresta equatorial úmida da estação experimental de silvicultura tropical do INPA. Acta Amazonica, 16/17: 411- 508.

Lamprecht, H. 1964. Ensayo sobre la estructura floristica de la parte sur-oriental del Bosque Universitario "El caimital”, Estado Barinas. Rev. For, Venez., 7(10-11): 77-119.

Mesquita, R.C.G. 2000. Management of advanced regeneration in secondary forests of the Brazilian Amazon. Forest Ecology and Management, 130: 131-140.

Montoya Maquin, J.M. 1966. El escuerdo de Yangambi (1956) como base para una nomeclatura de tipos de vegetación em el trópico americano. Turrialba, 16(12): 80-169.

Nelson, B.W.; Mesquita, R.; Pereira, J.L.G.; Souza, S.G.S.; Batista, G.T.; Couto, L. 1999. Allometric regressions for improved estimate of secondary forest biomass in the Central Amazon. Forest Ecology and Management, 117: 149-167.

Ranzani, G. 1980. Identificação e caracterização de alguns solos da Estação Experimental de Silvicultura Tropical do INPA. Acta Amazonica, 10(1): 7-41.

Saldarriga, J.G.; West, M.L.; Tharp, M.L.; Uhl, C. 1988. LongTerm chronosequence of forest succession in the upper Rio Negro of Colombia and Venezuela. Journal of Ecology, 76: 938958.

Salomão, R.P.; Nepstad, D.C.; Vieira, I.C. 1998. Biomassa e estoque de florestas tropicais primária e secundária. In: Floresta Amazônica: Dinâmica, Regeneração e Manejo (Gascon, C. e Moutinho, P. - editores). p. 99-119.

Uhl, C. 1982. Recovery following disturbances of different intensities in the Amazon rainforest of Venezuela. 7:19-24.

Recebido em 06/10/2006

Aceito em 07/02/2007 
